\title{
ASYMPTOTIC BEHAVIOR FOR A CLASS OF NONLOCAL NONAUTONOMOUS PROBLEMS
}

\author{
FLANK D. M. BEZERRA ${ }^{1}$, SEVERINO H. DA SILVA ${ }^{2}$, AND ANTÔNIO L. PEREIRA ${ }^{3}$
}

ABstRaCt. In this paper we consider the nonlocal nonautonomous evolution problem

$$
\left\{\begin{array}{l}
\partial_{t} u=-u+g(t, K u) \text { in } \Omega \\
u=0 \text { in } \mathbb{R}^{N} \backslash \Omega .
\end{array}\right.
$$

where $\Omega$ is a smooth bounded domain in $\mathbb{R}^{N}, g: \mathbb{R} \times \mathbb{R} \rightarrow \mathbb{R}$ and $K$ is an integral operator with a symmetric kernel. We prove existence and some regularity properties of the pullback attractor. We also show additional forward asymptotic results in the asymptotically autonomous case, using the properties of the Lyapunov functional for the limiting problem.

Mathematical Subject Classification 2010: 35B40, 35B41, 37B55.

Key words and phrases: Pullback attractors; nonlocal diffusion equations; nonautonomous equations; evolution process.

\section{INTRODUCTION}

The understanding of the dynamics of nonautonomous evolution equations has attracted the attention of many researchers in recent years; see for instance [4, 5, 6, 9, 12] and [14]. The results obtained here constitute an extension of the ones in [1] to our (more general) situation. We also prove new results in a different phase space $\left(L^{\infty}\right)$ and in the asymptotically autonomous case.

More precisely, we consider here the nonlocal nonautonomous evolution problem

$$
\text { prob }\left\{\begin{array}{l}
\partial_{t} u(t, x)=-u(t, x)+g(t, K u(t, x)) \text { for } t \geq \tau \in \mathbb{R} \text { and } x \in \Omega, \\
u(\tau, \cdot)=u_{\tau}(\cdot) \text { in } \Omega, \\
u(t, x)=0 \text { for } t \geq \tau \in \mathbb{R} \text { and } x \in \mathbb{R}^{N} \backslash \Omega .
\end{array}\right.
$$

where $\Omega$ is a bounded smooth domain in $\mathbb{R}^{N}(N \geq 1), g: \mathbb{R} \times \mathbb{R} \rightarrow \mathbb{R}$ is a (sufficiently smooth) function and $K$ is an integral operator with symmetric kernel

$$
K u(\cdot, x):=\int_{\mathbb{R}^{N}} J(x, y) u(\cdot, y) d y .
$$

We will suppose, without loss of generality, that $\int_{\mathbb{R}^{N}} J(x, y) d y=\int_{\mathbb{R}^{N}} J(x, y) d x=1$.

The paper is organized as follows. After recalling some concepts and results about attractors for infinite-dimensional nonautonomous dynamical systems in Section 2, we prove well

Date: April 16, 2018.

${ }^{2}$ Research partially supported by CAPES/CNPq. 
posedness of (1.1) in a Banach space which is isomorphic to $L^{p}(\Omega), 1 \leq p \leq \infty$, in Section 3, and existence and some regularity properties for the pullback attractor on Section 4, In Section 5 we prove some comparison results. In Section 6 is dedicated to the proof of upper semi-continuity of the family of pullback attractors with respect to the functional parameter $g$. Finally, on Section 17, we prove some additional results on the asymptotic behavior of (1.1) for some special cases, including the asymptotic autonomous one.

\section{SOME PRELIMINARY DEFINITIONS AND RESUlTS}

$\langle$ prelim $\rangle$ We start by recalling the concepts of evolution processes and pullback attractors which proved to be a useful tool to study the asymptotic dynamics of infinite dimensional nonautonomous dynamical system. We refer to [3, 4, [5, 6, 9] and [12] for more details and proofs.

Definition 2.1 (Nonlinear evolution process). A nonlinear evolution process in a Banach space $\mathcal{X}$ is a family of maps $\{S(t, \tau) ; t \geq \tau \in \mathbb{R}\}$ (not necessarily linear) from $\mathcal{X}$ into itself with the following properties:

(1) $S(t, t)=I$, for all $t \in \mathbb{R}$,

(2) $S(t, \tau)=S(t, s) S(s, \tau)$, for all $\tau \leq s \leq t$,

(3) The $\operatorname{map}\left\{(t, \tau) \in \mathbb{R}^{2} ; t \geq \tau\right\} \times \mathcal{X} \ni(t, \tau, x) \mapsto S(t, \tau) x \in \mathcal{X}$ is continuous.

In the particular case where each $S(t, \tau)$ is linear, $t \geq \tau \in \mathbb{R}$, we say that $\{S(t, \tau) ; t \geq \tau \in$ $\mathbb{R}\}$ is a linear process.

If $S(t, \tau)=S(t-\tau, 0)$ for all $t \geq \tau \in \mathbb{R}$, we say that the process is autonomous, and the family $\{\bar{S}(t)=S(t, 0) ; t \geq 0\}$ is then called a semigroup or a dynamical system.

Definition 2.2. The pullback (resp. forward) orbit of a subset $B$ of $\mathcal{X}$, at time $t \in \mathbb{R}$, is the set $\gamma_{p}(B, t):=\cup_{s \leq t} S(t, s) B\left(\right.$ resp. $\left.\gamma_{f}(B, t):=\cup_{s \geq t} S(s, t) B\right)$.

Definition 2.3. A globally-defined solution (or simply a global solution) of the evolution process $\{S(t, \tau) ; t \geq \tau \in \mathbb{R}\}$ through $\psi_{0} \in \mathcal{X}$ at time $\tau_{0}$ is a function $\psi: \mathbb{R} \rightarrow \mathcal{X}$ such that $\psi\left(\tau_{0}\right)=\psi_{0}$, and $S(t, \tau) \psi(\tau)=\psi(t)$, for all $t \geq \tau$. A global solution through $\psi_{0} \in \mathcal{X}$ is a global solution through $\psi_{0} \in \mathcal{X}$ at some time.

Definition 2.4 (Pullback Attraction). A family of sets $\{\mathcal{K}(t) ; t \in \mathbb{R}\}$ pullback attracts bounded subsets of $\mathcal{X}$ under $\{S(t, \tau) ; t \geq \tau \in \mathbb{R}\}$, at time $t$ if, for each bounded set $C \subset \mathcal{X}$,

$$
\lim _{\tau \rightarrow-\infty} \operatorname{dist}(S(t, \tau) C, \mathcal{K}(t))=0,
$$

where $\operatorname{dist}(\cdot, \cdot)$ denotes the Hausdorff semi-distance in $\mathcal{X}$,

$$
\operatorname{dist}(A, B)=\sup _{a \in A} \inf _{b \in B}|a-b|_{\mathcal{X}} .
$$

We observe that the Hausdorff semi-distance between $A$ and $B$, $\operatorname{dist}(A, B)$, examines how the set $A$ is contained in the set $B$. For example, $\operatorname{dist}(A, B)=0$ if and only if $A$ is contained in the closure of the set $B$.

$A$ family of sets $\{\mathcal{K}(t) ; t \in \mathbb{R}\}$ pullback attracts bounded subsets of $\mathcal{X}$ under $\{S(t, \tau) ; t \geq$ $\tau \in \mathbb{R}\}$ if it pullback attracts bounded subsets of $\mathcal{X}$ under $\{S(t, \tau) ; t \geq \tau \in \mathbb{R}\}$, at time $t$, for any $t$. 
Definition 2.5. A family of bounded subsets $\{B(t) ; t \in \mathbb{R}\}$ of $\mathcal{X}$ pullback absorbs bounded subsets of $\mathcal{X}$ under $\{S(t, \tau) ; t \geq \tau \in \mathbb{R}\}$ at time $t \in \mathbb{R}$. if, for any bounded set $C \subset \mathcal{X}$ and $\tau \leq t$, there exists $\tau_{0}(\tau, C) \in \mathbb{R}$ such that

$$
S(\tau, \tau-r) C \subset B(t), \text { for all } r \geq \tau_{0}(\tau, C) .
$$

$A$ family of bounded subsets $\{B(t) ; t \in \mathbb{R}\}$ of $\mathcal{X}$ pullback absorbs bounded subsets of $\mathcal{X}$ under $\{S(t, \tau) ; t \geq \tau \in \mathbb{R}\}$ if it pullback absorbs bounded subsets of $\mathcal{X}$ under $\{S(t, \tau) ; t \geq \tau \in \mathbb{R}\}$ at time $t$, for any $t \in \mathbb{R}$.

Remark 2.6. It is clear that, if $\{B(t) ; t \in \mathbb{R}\}$ pullback absorbs bounded subset of $\mathcal{X}$ at time $t$, then it pullback attracts bounded subsets of $\mathcal{X}$ at time $t$.

When $B(t)=B$, for all $\in \mathbb{R}$, where $B$ is some fixed bounded set, it is also said that the set $B$ (instead of the family $\{B(t) ; t \in \mathbb{R}\}$ ) pullback absorbs bounded subsets of $\mathcal{X}$.

Definition 2.7. An evolution process $\{S(t, \tau) ; t \geq \tau \in \mathbb{R}\}$ is said to be strongly pullback bounded dissipative if there exists a family of sets $\{\mathcal{K}(t) ; t \in \mathbb{R}\}$ that pullback attracts bounded subsets of $\mathcal{X}$ under $\{S(t, \tau)\}$ at time $s$ for any $s \leq t$.

Next, we present the notion of a set of pullback asymptotic states.

Definition 2.8 (Pullback attractor). A family $\{\mathcal{A}(t) ; t \in \mathbb{R}\}$ of compact subsets of $\mathcal{X}$ is said to be a pullback attractor for the evolution process $\{S(t, \tau) ; t \geq \tau \in \mathbb{R}\}$ if it is invariant, i.e., $S(t, \tau) \mathcal{A}(\tau)=\mathcal{A}(t)$ for all $\tau \leq t$, pullback attracts bounded subsets of $\mathcal{X}$, and is minimal, that is, if there is another family of closed sets $\{C(t) ; t \in \mathbb{R}\}$ which pullback attracts bounded subsets of $\mathcal{X}$, then $\mathcal{A}(t) \subset C(t)$, for all $t \in \mathbb{R}$.

Remark 2.9. The minimality requirement in the Definition 2.8 is an addition with respect to the theory of attractors for semigroups and is necessary to ensure uniqueness (see [4]). It can be dropped if we require that $\bigcup_{\tau \leq t} \mathcal{A}(\tau)$ is bounded for any $t \in \mathbb{R}$. In this case, we also have

$$
\mathcal{A}(t)=\{\xi(t): \xi: \mathbb{R} \rightarrow \mathcal{X} \text { is a global backwards bounded solution of } S(t, \tau)\},
$$

for all $t \in \mathbb{R}$.

Definition 2.10. An evolution process $\{S(t, \tau) ; t \geq \tau \in \mathbb{R}\}$ in a Banach space $\mathcal{X}$ is pullback asymptotically compact if, for each $t \in \mathbb{R}$, each sequence $\left\{\tau_{k}\right\}_{k \in \mathbb{N}}$ in $(-\infty, t]$ such that $\tau_{k} \rightarrow-\infty$ as $k \rightarrow \infty$, and each bounded sequence $\left\{z_{k}\right\}_{k \in \mathbb{N}}$ in $\mathcal{X}$ with $\left\{S\left(t, \tau_{k}\right) z_{k}\right\}_{k \in \mathbb{N}}$ bounded, the sequence $\left\{S\left(t, \tau_{k}\right) z_{k}\right\}_{k \in \mathbb{N}}$ possesses a convergent subsequence.

The following two results are proved in [4] (see also [5]) and will be used to prove the existence of the pullback attractor for the evolution process generated by (1.1), (see Section 4).

$\langle$ critcompact〉 Theorem 2.11. If an evolution process $\{S(t, \tau) ; t \geq \tau \in \mathbb{R}\}$ in a Banach space $\mathcal{X}$ satisfies the properties

$$
S(t, \tau)=T(t, \tau)+U(t, \tau), t \geq \tau
$$

where $U(t, \tau)$ is a compact operator and there exists a non-increasing function $k:[0,+\infty) \times$ $[0,+\infty) \rightarrow \mathbb{R}$ with $k(\sigma, r) \rightarrow 0$ as $\sigma \rightarrow+\infty$, and for all $\tau \leq t$ and $z \in \mathcal{X}$ with $|z|_{\mathcal{X}} \leq r$, 
$|T(t, \tau)|_{\mathcal{X}} \leq k(t-\tau, r)$, then the process $\{S(t, \tau) ; t \geq \tau \in \mathbb{R}\}$ is pullback asymptotically compact.

eorema 0.2.1 Theorem 2.12. If an evolution process $\{S(t, \tau) ; t \geq \tau \in \mathbb{R}\}$ in a Banach space $\mathcal{X}$ is strongly pullback bounded dissipative and pullback asymptotically compact, then $\{S(t, \tau) ; t \geq \tau \in \mathbb{R}\}$ possesses a pullback attractor $\{\mathcal{A}(t) ; t \in \mathbb{R}\}$. Moreover, the union $\bigcup_{\tau \leq t} \mathcal{A}(\tau)$ is bounded for each $t \in \mathbb{R}$, and the global pullback attractor is given by

$$
\mathcal{A}(t)=\Omega_{\wp}(\mathcal{B}(t), t):=\bigcap_{s \leq t} \overline{\bigcup_{\tau \leq s} S(t, \tau) \mathcal{B}(t)}, \text { for each } t \in \mathbb{R},
$$

where $\{B(t) ; t \in \mathbb{R}\}$ is a family of bounded subsets of $\mathcal{X}$ which pullback attracts bounded subsets of $\mathcal{X}$ at time $\tau$, for any $\tau \leq t$.

This theorem extends the analogous result for semigroups (cf. Theorem 1.1, Chapter 1 in [15]). The pullback attractor of strongly bounded dissipative process however, is always bounded in the past. To be more precise, for every $t \in \mathbb{R}$ the union $\bigcup_{\tau \leq t} \mathcal{A}(\tau)$ is bounded in $\mathcal{X}$.

\section{WELL POSEDNESS}

$\langle$ wellposed〉 In this section we show that the problem (1.1) is well posed in suitable phase spaces.

Consider for any $1 \leq p \leq \infty$ the subspace $X$ of $L^{p}\left(\mathbb{R}^{N}\right)$ given by

$$
X=\left\{u \in L^{p}\left(\mathbb{R}^{N}\right) ; u(x)=0, \text { if } x \in \mathbb{R}^{N} \backslash \Omega\right\}
$$

with the induced norm. The space $X$ is canonically isomorphic to $L^{p}(\Omega)$ and we usually identify the two spaces, without further comment. We also use the same notation for a function in $\mathbb{R}^{N}$ and its restriction to $\Omega$ for simplicity, wherever we believe the intention is clear from the context.

In order to obtain well posedness of (1.1), we consider the Cauchy problem

$$
\left\{\begin{array}{l}
\partial_{t} u=-u+F(t, u), \\
u(\tau)=u_{\tau}
\end{array}\right.
$$

where the map $F: \mathbb{R} \times X \rightarrow X$ is defined by

$$
F(t, u)(x)= \begin{cases}g(t, K u(t, x)), & x \in \Omega, \\ 0, & x \in \mathbb{R}^{N} \backslash \Omega .\end{cases}
$$

Definition 3.1. A solution of (3.1) in $[\tau, s), \tau<s$ is a continuous function $u:[\tau, s) \rightarrow X$ such that $u(\tau)=u_{\tau}$, the derivative with respect to $t$ exists and $\partial_{t} u(t, \cdot)$ belongs to $X$, and the differential equation in (3.1) is satisfied for $t \in[\tau, s)$.

The map given by

$$
K u(\cdot, x):=\int_{\mathbb{R}^{N}} J(x, y) u(\cdot, y) d y
$$


is well defined as a bounded linear operator in various function spaces, depending on the properties assumed for $J$. We collect here some estimates for this map which will be used in the sequel.

$\left\langle\right.$ boundK〉 Lemma 3.2. Let $K$ be the map defined by (3.3) and $\|J\|_{p}:=\sup _{x \in \Omega}\|J(x, \cdot)\|_{L^{p}(\Omega)}, 1 \leq p \leq$ $\infty$. If $u \in L^{p}(\Omega), 1 \leq p \leq \infty$, then $K u \in L^{p}(\Omega)$, and

(3.4) estimateL1

(3.5) estimateLq

$$
\begin{gathered}
\|K u\|_{L^{p}(\Omega)} \leq\|J\|_{1}\|u\|_{L^{p}(\Omega)}=\|u\|_{L^{p}(\Omega)} . \\
|K u(x)| \leq\|J\|_{q}\|u\|_{L^{p}(\Omega)}, \text { for all } x \in \Omega
\end{gathered}
$$

where $1 \leq q \leq \infty$ is the conjugate exponent of $p$.

Proof. Estimate (3.4) follows from Young's inequality and the fact that $\|J\|_{1}=1$ and estimate (3.5) follows from Hölder inequality.

$\langle$ loclip〉 Definition 3.3. If $E$ is a normed space, and $I \subset \mathbb{R}$ is an interval, we say that a function $F: I \times E \rightarrow E$ is locally Lipschitz continuous (or simply locally Lipschitz) in the second variable if, for any $\left(t_{0}, x_{0}\right) \in I \times E$, there exists a constant $C$ and a rectangle $R=\{(t, x) \in$ $\left.I \times E:\left|t-t_{0}\right|<a,\left\|x-x_{0}\right\|<b\right\}$ such that, if $(t, x)$ and $(t, y)$ belong to $R$ then $\| F(t, x)-$ $F(t, y)\|\leq C\| x-y \|$; we say that $F$ is Lipschitz continuous on bounded sets in the second variable if the rectangle $R$ in the previous definition can chosen as any bounded rectangle in $I \times E$

Remark 3.4. The two definitions in 3.3 are equivalent if the normed space $E$ is locally compact.

$\langle$ WellP $\rangle$ Proposition 3.5. Suppose, in addition to the hypotheses of Lemma 3.2, that the function $g$ is locally Lipschitz continuous in the second variable in $\mathbb{R} \times \mathbb{R}$. Then the function $F$ defined by (3.2) is bounded Lipschitz continuous in the second variable in $\mathbb{R} \times L^{p}(\Omega), 1 \leq p \leq \infty$.

Proof. Suppose $\left(t_{0}, u_{0}\right) \in \mathbb{R} \times X$. Then if $R$ is the rectangle $R:=\left\{(t, u) \in \mathbb{R} \times X|| t-t_{0} \mid<\right.$ $a,\left\|u-u_{0}\right\|_{L^{p}(\Omega)}<b$ it follows from (3.5) that $\left|K u_{0}(x)\right|<\|J\|_{q}\left\|u_{0}\right\|_{L^{p}(\Omega)},\left|K u(x)-K u_{0}(x)\right|<$ $\|J\|_{q} b$, for any $x \in \Omega$. Let $k_{R^{\prime}}$ be the Lipschitz constant of $g$ in the rectangle $R^{\prime}:=\{(t, x) \in$ $\left.I \times \mathbb{R}|| t-t_{0} \mid<a,\|x\| \leq\|J\|_{q}\left(\left\|u_{0}\right\|_{L^{p}(\Omega)}+b\right)\right\}$. Then, if $(t, u),(t, v) \in R$ we obtain $|g(t, K u(x))-g(t, K v(x))| \leq k_{R}^{\prime}|K u(x)-K v(x)|$ for any $x \in \Omega$. For $1 \leq p<\infty$, it follows then, from (3.4), that

$$
\begin{aligned}
\|g(t, K u)-g(t, K v)\|_{L^{p}(\Omega)} & \leq\left\{\int_{\Omega} k_{R^{\prime}}|K u(x)-K v(x)|^{p} d x\right\}^{1 / p} \\
& \left.=\left.k_{R^{\prime}}\left\{\int_{\Omega} \mid K(u-v)(x)\right)\right|^{p} d x\right\}^{1 / p} \\
& =k_{R^{\prime}}\|K(u-v)\|_{L^{p}(\Omega)} \\
& =k_{R^{\prime}}\|u-v\|_{L^{p}(\Omega)} .
\end{aligned}
$$

If $p=\infty$ the same inequality follows immediately from (3.4). Thus, the map

$$
u \in X \mapsto g(t, K u) \in X
$$


is Lipschitz in the rectangle $R$ and, therefore, so is the map $F(t, \cdot)$.

From the result above, it follows from well known results that the problem (3.1) has a local solution for any initial condition in $X$. For the global existence, we need the following result ([10] - Theorem 5.6.1)

$\left\langle\right.$ ladas〉 Theorem 3.6. Let $X$ be a Banach space, and suppose that $g:\left[t_{0}, \infty\right) \times X \rightarrow X$ is continuous and $\|g(t, u)\| \leq h(t,\|u\|) ;$ for all $(t, u) \in\left[t_{0}, \infty\right) \times X$, where $h:\left[t_{0}, \infty\right) \times \mathbb{R}^{+} \rightarrow \mathbb{R}^{+}$is continuous and $h(t, r)$ is non decreasing in $r \geq 0$, for each $t \in\left[t_{0}, \infty\right)$. Then, if the maximal solution $r\left(t, t_{0}, r_{0}\right)$ of the scalar initial value problem

$$
r^{\prime}=h(t, r), \quad r\left(t_{0}\right)=r_{0},
$$

exists throughout $\left[t_{0}, \infty\right)$, the maximal interval of existence of any solution $u\left(t, t_{0}, y_{0}\right)$ of the initial value problem

$$
\frac{d u}{d t}=g(t, u), \quad t \geq t_{0}, \quad u\left(t_{0}\right)=u_{0}
$$

also contains $\left[t_{0}, \infty\right)$.

〈globalexist〉 Corollary 3.7. Suppose, in addition to the hypotheses of Proposition 3.5, that $g$ satisfies:

$$
\limsup _{|x| \rightarrow \infty} \frac{|g(t, x)|}{|x|}<k_{1}, \text { for some constant } k_{1} \in \mathbb{R} .
$$

Then the problem (3.1) has a unique globally defined solution for any initial condition in $X$, which is given for $t \geq \tau$ by the "variation of constants formula"

$$
\begin{aligned}
& u\left(t, \tau, x ; u_{\tau}\right)=e^{-(t-\tau)} u_{\tau}(x)+\int_{\tau}^{t} e^{-(t-s)} F\left(s, u\left(s, \tau, x ; u_{\tau}\right)\right) d s \quad x \in \mathbb{R}^{N} \\
& \qquad \begin{array}{ll}
e^{-(t-\tau)} u_{\tau}(x)+\int_{\tau}^{t} e^{-(t-s)} g\left(s, K\left(u\left(s, \tau, \cdot ; u_{\tau}\right)\right)(x)\right) d s, & x \in \Omega, \\
0, & x \in \mathbb{R}^{N} \backslash \Omega .
\end{array}
\end{aligned}
$$

Proof. From Proposition 3.5, it follows that the right-hand-side of (3.1) is Lipschitz continuous in bounded sets of $X$ and, therefore, the Cauchy problem (3.1) is well posed in $X$ with a unique local solution $u\left(t, \tau, x ; u_{\tau}\right)$, given by (3.7) (see [7]).

From condition (3.6), it follows that there is a constant $k_{2}$, such that

(3.8) dissip2

$$
\|g(t, x)\| \leq k_{2}+k_{1}|x|, \quad \text { for any } \quad x \in \mathbb{R} .
$$

If $1 \leq p<\infty$, we obtain from (3.8) and (3.4) that

$$
\begin{aligned}
\|g(t, K u)\|_{L^{p}(\Omega)} & \leq k_{2}|\Omega|^{1 / p}+k_{1}\|K u\|_{L^{p}(\Omega)} \\
& \leq k_{2}|\Omega|^{1 / p}+k_{1}\|u\|_{L^{p}(\Omega)}
\end{aligned}
$$

For $p=\infty$, we obtain by the same arguments (or by making $p \rightarrow \infty$ ), that

$$
\|g(t, K u)\|_{L^{\infty}(\Omega)} \leq k_{2}+k_{1}\|u\|_{L^{\infty}(\Omega)} .
$$


Defining $h:\left[t_{0}, \infty\right) \times \mathbb{R}^{+} \rightarrow \mathbb{R}^{+}$, by $h(t, r)=k_{2}|\Omega|^{1 / p}+\left(k_{1}+1\right) r$, it follows that Problem 3.1 satisfies the hypothesis of Theorem 3.6 and the global existence follows immediately. The variation of constants formula can be verified by direct derivation.

The result below can be found in [13].

〈Prop-Rall〉 Proposition 3.8. Let $Y$ and $Z$ be normed linear spaces, $F: Y \rightarrow Z$ a map and suppose that the Gateaux derivative of $F, D F: Y \rightarrow \mathcal{L}(Y, Z)$ exists and is continuous at $y \in Y$. Then the Frechèt derivative $F^{\prime}$ of $F$ exists and is continuous at $y$.

$\langle\mathrm{C} 1 \mathrm{flow}\rangle$ Proposition 3.9. Suppose, in addition to the hypotheses of Corollary 3.7 that the function $g$ is continuously differentiable on $\mathbb{R} \times \mathbb{R}$. Then, for each $t \in \mathbb{R}$ the function $F(t, \cdot)$ is continuously Frechét differentiable on $X$ with derivative given by

$$
D F(t, u) v(x):= \begin{cases}D_{2} g(t, K u(t, x)) K v(t, x), & x \in \Omega, \\ 0, & x \in \mathbb{R}^{N} \backslash \Omega .\end{cases}
$$

where $D_{2} g(t, y)=\partial_{y} g$ stands for the partial derivative of $g$ with respect to the second variable.

Proof. From a simple computation, using the fact $g$ is continuously differentiable on $\mathbb{R}$, it follows that the Gateaux's derivative of $F(t, \cdot)$ is given by

$$
D F(t, u) v(x):= \begin{cases}D_{2} g(t, K u(t, x)) K v(t, x), & x \in \Omega, \\ 0, & x \in \mathbb{R}^{N} \backslash \Omega .\end{cases}
$$

The operator $D F(t, u)$ is clearly a linear operator in $X$. Using estimate (3.5) and the continuity of $D_{2} g$ it follows that, for any $t \in \mathbb{R}$ and $u \in X, D_{2} g(t, K u(t, \cdot))$ is bounded by a constant $k_{3}$. Using (3.4), we obtain

$$
\begin{aligned}
\|D F(t, u) v\|_{X} & \leq k_{3}\|K v\|_{L^{p}(\Omega)} \\
& \leq k_{3}\|v\|_{X},
\end{aligned}
$$

proving that $D F(t, \cdot)$ is a bounded operator.

We now prove that $D F(t, \cdot): X \rightarrow \mathcal{L}(X)$ is continuous. If $w$ and $u$ in $X$, it follows from (3.5) that

$$
\begin{aligned}
& \|D F(t, u) v-D F(t, w) v\|_{L^{p}(\Omega)} \\
\leq & \left\{\int_{\Omega}\left|D_{2} g(t, K u(x))-D_{2} g(t, K w(x))\right|^{p}|K v(x)|^{p} d x\right\}^{1 / p} \\
\leq & \|J\|_{q}\left\{\int_{\Omega}\left|D_{2} g(y, K u(x))-D_{2} g(t, K w(x))\right|^{p} d x\right\}^{1 / p}\|v\|_{L^{p}(\Omega)} .
\end{aligned}
$$

Now, from (3.5), it follows that $K u(x)$ and $K w(x)$ are in a bounded set of $\mathbb{R}^{N}$ for $w$ in a neighborhood of $u$ in $L^{p}(\Omega)$ and any $t \in \mathbb{R}, x \in \Omega$. Also, $|K u(x)-K w(x)| \rightarrow 0$ uniformly in $\Omega$, as $w \rightarrow u$ in $L^{p}(\Omega)$. Therefore, $\left|D_{2} g(t, K u(x))-D_{2} g(t, K w(x))\right| \rightarrow 0$ uniformly in $\Omega$, by the continuity of $D_{2} g$. From this the continuity of $D F(t, \cdot)$ at $u$ follows immediately.

Hence, it follows from Proposition 3.8 that $F(t, \cdot)$ is Frechet differentiable with continuous derivative in $X$. 
Remark 3.10. Since the right-hand side of (3.1) is a $C^{1}$ function, the process generated by (3.1) in $X$ is $C^{1}$ with respect to initial conditions.

From the results above, we have that, for each $\tau \in \mathbb{R}$ and $u(\tau, \cdot) \in X$, the unique solution of (3.1) with initial condition $u(\tau, \cdot)$ exists for all $t \geq \tau$ and this solution $(t, x) \mapsto u\left(t, \tau, x ; u_{\tau}\right)$ (defined by (3.7)) gives rise to a family of nonlinear $C^{1}$ evolution process on $X$ given by

$$
S(t, \tau) u_{\tau}(x):=u\left(t, \tau, x ; u_{\tau}\right), t \geq \tau \in \mathbb{R}, x \in \mathbb{R}^{N} .
$$

\section{Existence AND Regularity of the PUllBaCK ATtractor}

LlAttractors $\quad$ We prove the existence of a pullback attractor $\{\mathcal{A}(t) ; t \in \mathbb{R}\}$ in $X$ for the evolution process $\{S(t, \tau) ; t \geq \tau \in \mathbb{R}\}$ when $1 \leq p \leq \infty$. In the estimates below the constant, $|\Omega|^{\frac{1}{p}}$ should be taken as 1 when $p=\infty$.

$\left\langle\mathrm{L}_{-} \mathrm{PullAbs}\right\rangle$ Lemma 4.1. Suppose that the hypotheses of Proposition 3.9 hold with the constant $k_{1}$ in (3.8) satisfying $k_{1}<1$ Then the ball of $L^{p}(\Omega), 1 \leq p \leq \infty$, centered at the origin with radius $(1+\delta) \frac{k_{2}|\Omega|^{\frac{1}{p}}}{1-k_{1}}$, where $k_{1}$ and $k_{2}$ are the constants appearing in (3.8) and $\delta$ is any positive number pullback absorbs bounded subsets of $X$ under the evolution process $\{S(t, \tau) ; t \geq \tau \in \mathbb{R}\}$ generated by (3.1) (with $|\Omega|^{\frac{1}{p}}$ replaced by 1 if $p=\infty$ ).

Proof. If $u\left(t, \tau, x ; u_{\tau}\right)$ is a solution of (3.1) with initial condition $u_{\tau}$ then, for $1 \leq p<\infty$

$$
\begin{aligned}
\frac{d}{d t} \int_{\Omega}\left|u\left(t, \tau, x ; u_{\tau}\right)\right|^{p} d x & =\frac{d}{d t} \int_{\Omega}\left|u\left(t, \tau, x ; u_{\tau}\right)\right|^{p} d x \\
& =\int_{\Omega} p|u|^{p-1} \operatorname{sgn}(u) u_{t}\left(t, \tau, x ; u_{\tau}\right) d x \\
& =-p \int_{\Omega}|u|^{p}\left(t, \tau, x ; u_{\tau}\right) d x+p \int_{\Omega}|u|^{p-1} \operatorname{sgn}(u) g(t, K u) d x .
\end{aligned}
$$

Using Hölder's inequality, condition (3.6) and estimate (3.5), we obtain

$$
\begin{aligned}
\int_{\Omega}|u|^{p-1} \operatorname{sgn}(u) g(t, K u) d x & \leq\left(\left\|u\left(t, \tau, \cdot ; u_{\tau}\right)\right\|_{L^{p}(\Omega)}^{(p-1) q}\right)^{\frac{1}{q}}\left(\int_{\Omega}|g(t, K u)|^{p} d x\right)^{\frac{1}{p}} \\
& \leq\left(\left\|u\left(t, \tau, \cdot ; u_{\tau}\right)\right\|_{L^{p}(\Omega)}^{p}\right)^{\frac{p-1}{p}}\left(\int_{\Omega}\left(k_{1}|K u|+k_{2}\right)^{p} d x\right)^{\frac{1}{p}} \\
& \leq\left\|u\left(t, \tau, \cdot ; u_{\tau}\right)\right\|_{L^{p}(\Omega)}^{p-1}\left[k_{1}\left\|u\left(t, \tau, \cdot ; u_{\tau}\right)\right\|_{L^{p}(\Omega)}+k_{2}|\Omega|^{\frac{1}{p}},\right]
\end{aligned}
$$

where $q$ is the conjugate exponent of $p$.

Hence

$$
\begin{aligned}
\frac{d}{d t}\left\|u\left(t, \tau, \cdot ; u_{\tau}\right)\right\|_{L^{p}(\Omega)}^{p} & \leq-p\left\|u\left(t, \tau, \cdot ; u_{\tau}\right)\right\|_{L^{p}(\Omega)}^{p}+p k_{1}\left\|u\left(t, \tau, \cdot ; u_{\tau}\right)\right\|_{L^{p}(\Omega)}^{p} \\
& +p k_{2}|\Omega|^{\frac{1}{p}}\left\|u\left(t, \tau, \cdot ; u_{\tau}\right)\right\|_{L^{p}(\Omega)}^{p-1} \\
& =p\left\|u\left(t, \tau, \cdot ; u_{\tau}\right)\right\|_{L^{p}(\Omega)}^{p}\left[-1+k_{1}+\frac{k_{2}|\Omega|^{\frac{1}{p}}}{\left\|u\left(t, \tau, \cdot ; u_{\tau}\right)\right\|_{L^{p}(\Omega)}}\right] .
\end{aligned}
$$


Since $k_{1}<1, \varepsilon=1-k_{1}>0$. Thus, while $\left\|u\left(t, \tau, \cdot ; u_{\tau}\right)\right\|_{L^{p}(\Omega)} \geq(1+\delta) \frac{k_{2}|\Omega|^{\frac{1}{p}}}{\varepsilon}$, we have

$$
\begin{aligned}
\frac{d}{d t}\left\|u\left(t, \tau, \cdot ; u_{\tau}\right)\right\|_{L^{p}(\Omega)}^{p} & \leq p\left\|u\left(t, \tau, \cdot ; u_{\tau}\right)\right\|_{L^{p}(\Omega)}^{p}\left(-\varepsilon+\frac{\varepsilon}{1+\delta}\right) \\
& =-p \frac{\delta}{1+\delta} \varepsilon\left\|u\left(t, \tau, \cdot ; u_{\tau}\right)\right\|_{L^{p}(\Omega)}^{p} .
\end{aligned}
$$

Therefore, while $\left\|u\left(t, \tau, \cdot ; u_{\tau}\right)\right\|_{L^{p}(\Omega)} \geq(1+\delta) \frac{k_{2}|\Omega|^{\frac{1}{p}}}{1-k_{1}}$, we have

(4.1) boundsol

$$
\begin{aligned}
\left\|u\left(t, \tau, \cdot ; u_{\tau}\right)\right\|_{L^{p}(\Omega)}^{p} & \leq e^{-\frac{\varepsilon \delta p}{(1+\delta)} t}\left\|u_{\tau}\right\|_{L^{p}(\Omega)}^{p} \\
& =e^{-\frac{\delta p}{(1+\delta)}\left(1-k_{1}\right) t}\left\|u_{\tau}\right\|_{L^{p}(\Omega)}^{p} .
\end{aligned}
$$

From this, the result follows easily for $1 \leq p \leq \infty$. Since the estimates are uniform in $p$, it also follows for $p=\infty$, by taking the limit with $p \rightarrow \infty$.

$\left\langle\right.$ Theor1〉 Theorem 4.2. In addition to the conditions of Lemma 4.1, suppose that $\left\|J_{x}(x, \cdot)\right\|_{L^{q}(\Omega)}$ is bounded, where $q$ is the conjugate exponent of $p$ and $D_{2} g(t, \cdot)$ the derivative of $g$ with respect to the second variable is bounded in bounded sets of $\mathbb{R}$, uniformly for $t \in \mathbb{R}$. Then there exists a pullback attractor $\{\mathcal{A}(t) ; t \in \mathbb{R}\}$ for the evolution process $\{S(t, \tau) ; t \geq \tau \in \mathbb{R}\}$ generated by (3.1) in $X=L^{p}(\Omega)$ and $\mathcal{A}(t)$ is contained in the ball of radius $\frac{k_{2}|\Omega|^{\frac{1}{p}}}{1-k_{1}}$ in $L^{p}(\Omega)$, for any $t \in \mathbb{R}$ and $1 \leq p \leq \infty$.

Proof. From (3.7), it follows that

$$
S(t, \tau) u_{\tau}=T(t, \tau) u_{\tau}+U(t, \tau) u_{\tau}, t \geq \tau, x \in \Omega
$$

where

$$
T(t, \tau) u_{\tau}(x):=e^{-(t-\tau)} u_{\tau}(x)
$$

and

$$
U(t, \tau) u_{\tau}(x):=\int_{\tau}^{t} e^{-(t-s)} g\left(s, K u\left(s, \tau, x ; u_{\tau}\right)\right) d s .
$$

Suppose $u_{\tau} \in B$, where $B$ is a bounded subset of $X$. We may suppose that $B$ is contained in the ball centered at the origin of radius $r>0$. Then

$$
\left\|T(t, \tau) u_{\tau}\right\|_{L^{p}(\Omega)} \leq r e^{-(t-\tau)}, t \geq \tau \in \mathbb{R}
$$

Also, from (4.1), we have that $\left\|u\left(t, \tau, \cdot ; u_{\tau}\right)\right\|_{L^{p}(\Omega)} \leq M$, for $t \geq \tau$, where $M=\max \left\{r, \frac{2 k_{2}|\Omega| \frac{1}{p}}{1-k_{1}}\right\}$. Hence, for all $t \geq \tau$, and $x \in \Omega$, we obtain

$$
\left|\partial_{x} U(t, \tau) u(\tau, x)\right| \leq \int_{\tau}^{t} e^{-(t-s)}\left|D_{2} g\left(s, K u\left(s, \tau, x ; u_{\tau}\right)\right)\right|\left|\partial_{x} K u\left(s, \tau, x ; u_{\tau}\right)\right| d s .
$$

By Hölder's inequality we have that

(4.2) Est-No

$$
\begin{aligned}
\left|\partial_{x} K u\left(s, \tau, x ; u_{\tau}\right)\right| & \leq \int_{\Omega}\left|\partial_{x} J(x, y) \| u\left(s, \tau, y ; u_{\tau}\right)\right| d y \\
& \leq\left\|\partial_{x} J(x, \cdot)\right\|_{L^{q}(\Omega)}\left\|u\left(s, \tau, \cdot ; u_{\tau}\right)\right\|_{L^{p}(\Omega)} \\
& \leq C\left\|u\left(s, \tau, \cdot ; u_{\tau}\right)\right\|_{L^{p}(\Omega)}
\end{aligned}
$$


for some $C>0$ such that $\sup _{x \in \Omega}\left\|\partial_{x} J(x, \cdot)\right\|_{L^{q}(\Omega)} \leq C$. Since $\left\|u\left(t, \tau, \cdot ; u_{\tau}\right)\right\|_{L^{p}(\Omega)} \leq M$, for $t \geq \tau$, we obtain, using estimate (3.5) that $K u\left(t, \tau, \cdot ; u_{\tau}\right)$ is bounded by a constant independent of $t \geq \tau$ and $u_{\tau} \in B$. Using the hypotheses, it follows that $D_{2} g\left(t, K u\left(t, \tau, \cdot ; u_{\tau}\right)\right)$ is bounded by a constant $k_{3}$ for any $t \geq \tau$ and $u_{\tau} \in B$. Thus

$$
\begin{aligned}
\left|\partial_{x} U(t, \tau) u_{\tau}(x)\right| & \leq k_{3} C M \int_{\tau}^{t} e^{-(t-s)} d s \\
& \leq k_{3} C M
\end{aligned}
$$

Therefore, for $t \geq \tau$ and any $u_{\tau} \in B$, the value of $\left\|\partial_{x} U(t, \tau) u_{\tau}\right\|_{L^{p}(\Omega)}$ is bounded by a constant (independent of $\tau$ and $u_{\tau} \in B$ ). It follows that for all $u_{\tau} \in B$, we have that $U(t, \tau) u_{\tau}$ belongs to a (fixed) ball of $W^{1, p}(\Omega)$ for all $u_{\tau} \in B$. From Sobolev's Embedding Theorem, it follows that $U(t, \tau)$ is a compact operator, for any $\tau \leq t$ and then, from Theorem 2.11, it follows that the process $\{S(t, \tau) ; t \geq \tau \in \mathbb{R}\}$ is asymptotically compact.

Therefore, it follows from Lemma 4.1 and Theorem 2.12 that the pullback attractor $\{\mathcal{A}(t) ; t \in \mathbb{R}\}$ exists and is the pullback $\Omega$-limit set of any bounded subset of $X$ containing $\mathcal{B}_{\delta}$, where $\mathcal{B}_{\delta}=B\left(0, \frac{(1+\delta) k_{2}|\Omega|^{\frac{1}{p}}}{1-k_{1}}\right)$, for any $\delta>0$ i.e., for instance

$$
\mathcal{A}(t)=\Omega_{\wp}\left(\mathcal{B}_{\delta}, t\right):=\bigcap_{s \leq t} \overline{\bigcup_{\tau \leq s} S(t, \tau) \mathcal{B}_{\delta}}, \quad \text { for each } t \in \mathbb{R} .
$$

From this, since $\mathcal{B}_{\delta}$ pullback absorbs bounded subsets of $X$, it also follows that $\mathcal{A}(t)$ is contained in the ball centered at the origin of radius $\frac{k_{2}|\Omega|^{\frac{1}{p}}}{1-k_{1}\|\beta\|_{L^{\infty}(\mathbb{R})}}$ in $L^{p}\left(\mathbb{R}^{N}\right)$, for any $t \in \mathbb{R}$ $1 \leq p \leq \infty$.

Theorem 4.3. Assume the same conditions as in Lemma 4.1. Then there exists a bounded set of $W^{1, p}(\Omega), 1 \leq p \leq \infty$ containing $\mathcal{A}(t)$, for any $t \in \mathbb{R}$.

Proof. From Theorem 4.2, we obtain that $\mathcal{A}(t)$ is contained in the ball centered at the origin and radius $\frac{k_{2}|\Omega|^{\frac{1}{p}}}{1-k_{1}}$ in $L^{p}(\Omega)$. Furthermore, from Theorem 4.2 , we get that the pullback attractor can be written as the set of all global bounded solutions. Hence, if $u\left(t, \tau, x ; u_{\tau}\right)$ is a solution of (3.1) such that $u\left(t, \tau, x ; u_{\tau}\right) \in \mathcal{A}(t)$ for all $t \in \mathbb{R}$, then

$$
\text { ?equality_Lp? } u\left(t, \tau, x ; u_{\tau}\right)=\int_{-\infty}^{t} e^{-(t-s)} g\left(s, K u\left(s, \tau, x ; u_{\tau}\right)\right) d s,
$$

where the equality above is in the sense of $L^{p}\left(\mathbb{R}^{N}\right)$.

Proceeding as in the proof of the Theorem 4.2 (see the estimate (4.2) above), we have that

$$
\begin{aligned}
\left|\partial_{x} u\left(t, \tau, x ; u_{\tau}\right)\right| & \leq \int_{-\infty}^{t} e^{-(t-s)}\left|D_{2} g\left(s, K u\left(s, \tau, x ; u_{\tau}\right)\right)\right|\left|\partial_{x} K u\left(s, \tau, x ; u_{\tau}\right)\right| d s \\
& \leq k_{3} C M .
\end{aligned}
$$

for some $C>0$ such that $\sup _{x \in \Omega}\left\|\partial_{x} J(x, \cdot)\right\|_{L^{q}(\Omega)} \leq C$, and $k_{3}$ is a bound of $\left|D_{2} g(t, K u(\cdot))\right|$ for $u$ in the ball of radius $\frac{2 k_{2}|\Omega|^{\frac{1}{p}}}{1-k_{1}}$ in $L^{p}(\Omega)$. It follows that $\mathcal{A}(t)=S(t, \tau) \mathcal{A}(\tau)$ is in a bounded set of $W^{1, p}(\Omega)$ uniformly for $t \in \mathbb{R}$. 


\section{A COMPARISON RESULT}

〈comparison〉 In this section we prove a comparison result in $L^{\infty}\left(\mathbb{R}^{N}\right)$ and use it to prove the invariance of some sets under $S(t, \tau)$.

Definition 5.1. A function $v=v\left(t, \tau, x ; v_{\tau}\right)$ is a sub solution of the Cauchy problem (3.1) with initial condition $v_{\tau}$ in $L^{\infty}\left(\mathbb{R}^{N}\right)$ if $v(\tau, x)=v_{\tau}(x)$ for almost everywhere (a.e.) $x \in \Omega, v$ is continuously differentiable with respect to $t$ and satisfies

$$
\partial_{t} v \leq-v+g(t, K v)
$$

a. e. in $[\tau,+\infty] \times \Omega$.

Analogously, the function $V\left(t, \tau, x ; u_{\tau}\right)$ is a super solution of (3.1) with initial condition $V_{\tau}$ if $V(\tau, x)=V_{\tau}(x)$, for almost everywhere (a.e.) $x \in \Omega, V$ is continuously differentiable with respect to $t$ and satisfies

$$
-V+g(t, K V) \leq \partial_{t} V .
$$

〈Comparation〉 Theorem 5.2. Assume the functions $f, g$ and $h$ satisfy the same conditions required for $g$ in Proposition 3.9. Assume also that $f(t, x) \leq g(t, x) \leq h(t, x)$, a.e. for $(t, x) \in \mathbb{R} \times \mathbb{R}$ and the functions $f(t, \cdot)$ and $h(t, \cdot)$ are increasing. Let $v\left(t, \tau, x ; u_{\tau}\right),\left(V\left(t, \tau, x ; u_{\tau}\right)\right)$ be a sub solution (super solution) of the Cauchy problem (3.1) with $f(h)$ substituted for $g$ and initial condition $v_{\tau}\left(V_{\tau}\right) \in L^{\infty}(\Omega)$ and $u\left(t, \tau, x ; u_{\tau}\right)$ a solution of (3.1), with initial condition $u_{\tau} \in L^{\infty}(\Omega)$. Then, if $v(\tau, \cdot) \leq u(\tau, \cdot) \leq V(\tau, \cdot)$ a.e. in $\Omega$.

$$
v\left(t, \tau, x ; v_{\tau}\right) \leq u\left(t, \tau, x ; u_{\tau}\right) \leq V\left(t, \tau, x ; V_{\tau}\right) \text { a.e. in }[\tau,+\infty] \times \Omega .
$$

Proof. Consider the operator $G$ on $L^{\infty}([\tau, T] \times \Omega)$, for some $T \in \mathbb{R}$ (to be fixed later), defined by

$$
\operatorname{def} \phi)(t, x)= \begin{cases}\left.e^{-(t-\tau)} \phi(\tau, x)+\int_{\tau}^{t} e^{-(t-s)} g(s, K \phi)(s, x)\right) d s, & x \in \Omega, \\ 0, & x \in \mathbb{R}^{N} \backslash \Omega .\end{cases}
$$

If $T>\tau$, it follows from (3.5) that $|K \phi(t, x)| \leq\|\phi\|_{L^{\infty}([\tau, T] \times \Omega)}$ and, therefore, $|g(t, K \phi(t, x))|$ is bounded by a constant $b$.

Thus, for any $(t, x) \in[\tau, T] \times \Omega$

$$
\begin{aligned}
|(G \phi)(t, x)| & \leq e^{-(t-\tau)}|\phi(\tau, x)|+\int_{\tau}^{t} e^{-(t-s)}|g(s, K \phi(s, x))| d s \\
& \leq e^{-(t-\tau)}|\phi(\tau, x)|+b \int_{\tau}^{t} e^{-(t-s)} d s \\
& \leq\|\phi(\tau, \cdot)\|_{L^{\infty}(\Omega)}+b(T-\tau)
\end{aligned}
$$

Therefore $G$ is well defined as an operator on $L^{\infty}([\tau, T] \times \Omega)$ and furthermore $(G \phi)(\tau, x)=$ $\phi(\tau, x)$.

Let $u_{\tau} \in L^{\infty}(\Omega), L \in \mathbb{R}$ a positive number, and consider the set $\mathfrak{M}_{\mathfrak{u}_{\tau}}$ in $L^{\infty}([\tau, T] \times \Omega)$ given by

$$
\mathfrak{M}_{u_{\tau}}=\left\{\phi ; \phi(\tau, x)=u_{\tau}(x) \text { and }\left|\phi(t, x)-u_{\tau}(x)\right| \leq L, \text { for } \tau \leq t \leq T\right\} \text {. }
$$


if $\phi \in \mathfrak{M}_{u_{\tau}}$ then, from (3.5), it follows that, for all $(t, x) \in[\tau, T] \times \Omega$ and $\phi \in \mathfrak{M}_{u_{\tau}}$

$$
\text { estimateKphi }|K \phi(t, x)| \leq\|\phi\|_{L^{\infty}([\tau, T] \times \Omega)} \leq\left(L+\left\|u_{\tau}\right\|_{L^{\infty}(\Omega)}\right)
$$

and, therefore, $|g(t, K \phi(t, x))|$ is bounded by a constant $b$ for $(t, x) \in[\tau, T] \times \Omega$ and $\phi \in \mathfrak{M}_{u_{\tau}}$. Thus, for any $(t, x) \in[\tau, T] \times \Omega$

$$
\begin{aligned}
\left|(G \phi)(t, x)-u_{\tau}(x)\right| & \leq\left(1-e^{-(t-\tau)}\right)\left|u_{\tau}(x)\right|+\int_{\tau}^{t} e^{-(t-s)}|g(s, K \phi(s, x))| d s \\
& \leq\left(1-e^{-(t-\tau)}\right)\left|u_{\tau}(x)\right|+b \int_{\tau}^{t} e^{-(t-s)} d s \\
& \leq\left(1-e^{-(t-\tau)}\right)\left\|u_{\tau}\right\|_{L^{\infty}(\Omega)}+b(T-\tau) .
\end{aligned}
$$

and $(G \phi)(t, x)$ belongs to $\mathfrak{M}_{u_{\tau}}$, for any $\phi \in \mathfrak{M}_{u_{\tau}}$ if $(T-\tau)$ is small enough.

We now prove that $G$ is a contraction in $\mathfrak{M}_{u_{\tau}}$ for some $T>\tau$. Using (5.2) and the fact that $g$ is locally Lipschitz in the second variable, we obtain the existence of a constant $k_{\mathfrak{M}}$, such that, for $\phi_{1}, \phi_{2} \in \mathfrak{M}_{u_{\tau}}$

$$
\begin{aligned}
\left|g\left(t, K \phi_{1}(t, x)\right)-g\left(t, K \phi_{2}(t, x)\right)\right| & \leq k_{\mathfrak{M}}\left|K \phi_{1}(t, x)-K \phi_{2}(t, x)\right| \\
& \leq k_{\mathfrak{M}}\left|\phi_{1}(t, x)-\phi_{2}(t, x)\right| .
\end{aligned}
$$

Therefore

$$
\begin{aligned}
\mid\left(G \phi_{1}\right)(t, x) & -\left(G \phi_{2}\right)(t, x) \mid \\
& \leq \int_{\tau}^{t} e^{-(t-s)}\left|g\left(t, K \phi_{1}(s, x)\right)-g\left(s, K \phi_{2}(s, x)\right)\right| d s \\
& \leq k_{\mathfrak{M}} \int_{\tau}^{t} e^{-(t-s)}\left|\phi_{1}(s, x)-\phi_{2}(s, x)\right| d s \\
& \leq k_{\mathfrak{M}}\left\|\phi_{1}-\phi_{2}\right\|_{L^{\infty}([\tau, T] \times \Omega)} \int_{\tau}^{t} e^{-(t-s)} d s \\
& \leq k_{\mathfrak{M}}(T-\tau)\left\|\phi_{1}-\phi_{2}\right\|_{L^{\infty}([\tau, T] \times \Omega)} .
\end{aligned}
$$

Hence $G$ is a contraction in $\mathfrak{M}_{u_{\tau}}$ for $T-\tau$ small enough.

If $\phi(t, x)$ belongs to $\mathfrak{M}_{u_{\tau}}$ and $z(t, x)=\lim _{n \rightarrow \infty}\left(G^{n} \phi\right)(t, x)$ then, from the continuity of $G$, we obtain that

$$
(G z)(t, x)=G\left(\lim _{n \rightarrow \infty}\left(G^{n} \phi\right)(t, x)\right)=\lim _{n \rightarrow \infty}\left(G^{n+1} \phi\right)(t, x)=z(t, x) .
$$

Therefore $z(t, x)$ is (the unique) fixed point of $G$ in $\mathfrak{M}_{u_{\tau}}$ and it follows that $z(t, x)=$ $u\left(t, \tau, x ; u_{\tau}\right)$, the unique solution of (3.1) in $[\tau, T] \times \Omega$ with initial condition $z(\tau, x)=u_{\tau}(x)$. In particular

$$
u\left(t, \tau, x ; u_{\tau}\right)=\lim _{n \rightarrow \infty}\left(G^{n} u_{\tau}\right)(t, x) \text { in } L^{\infty}([\tau, T] \times \Omega),
$$

where, by an abuse of notation, we still denote by $u_{\tau}$ the function in $\mathfrak{M}_{u_{\tau}}$, which is equal to $u_{\tau}$ for $\tau \leq t \leq T$.

Let $F$ and $H$ defined as in (5.1) with $g$ replaced by $f$ or $h$, respectively. The same results proved above for $G$ remain true for $F$ and $H$ and furthermore, since $g$ and $h$ are increasing it follows that $F$ and $G$ are now monotonic, that is, for any $\phi_{1}, \phi_{2} \in L^{\infty}([\tau, T] \times \Omega)$ with 
$\phi_{1} \leq \phi_{2}$ a.e. in $[\tau, T] \times \Omega$, we have $F\left(\phi_{1}\right) \leq F\left(\phi_{2}\right)$ and $H\left(\phi_{1}\right) \leq H\left(\phi_{2}\right)$ a.e. in $[\tau, T] \times \Omega$ (for some $T>\tau)$.

Now, if $v$ is a sub solution of (3.1), with $g$ replaced by $f$ and initial condition $v_{\tau} \leq u_{\tau}$ a.e., we obtain

$$
v\left(t, \tau, x ; v_{\tau}\right) \leq e^{-(t-\tau)} v(\tau, x)+\int_{\tau}^{t} e^{-(t-s)} f\left(s, K v\left(s, \tau, x ; v_{\tau}\right)\right) d s \text { a.e. in }[\tau, T] \times \Omega .
$$

Hence

$$
v\left(t, \tau, x ; v_{\tau}\right) \leq(F v)\left(t, \tau, x ; u_{\tau}\right) \text { a.e. in }[\tau, T] \times \Omega \text {. }
$$

and it follows that $v\left(t, \tau, x ; v_{\tau}\right) \leq\left(F^{n} v\right)\left(t, \tau, x ; v_{\tau}\right)$ a.e. in $[\tau, T] \times \Omega$. Thus, passing to the limit as above, with an eventually smaller $T$ to ensure that $v\left(t, \tau, x ; v_{\tau}\right)$ belongs to $\mathfrak{M}_{v_{\tau}}$ and $H$ is a contraction in $\mathfrak{M}_{v_{\tau}}$ we obtain

$$
\text { comparesub } v\left(t, \tau, x ; v_{\tau}\right) \leq w\left(t, \tau, x ; v_{\tau}\right) \text {, a.e. in }[\tau, T] \times \Omega
$$

where $w\left(t, \tau, x ; v_{\tau}\right)$ is the unique solution of (3.1) in $[\tau, T] \times \Omega$ with initial condition $v_{\tau}$, and $f$ substituted for $g$.

Since $v(\tau, x) \leq u(\tau, x)$ a.e. in $\Omega$, and $f \leq g$ it follows that

$$
\left(F^{n} v_{\tau}\right)(t, x) \leq\left(G^{n} u_{\tau}\right)(t, x) \text { a.e. in }[\tau, T] \times \Omega .
$$

Passing to the limit as $n$ goes to infinity, we obtain

$$
w\left(t, \tau, x ; v_{\tau}\right) \leq u\left(t, \tau, x ; u_{\tau}\right) \text {, a.e. in }[\tau, T] \times \Omega .
$$

From (5.3) and (5.4) it follows that

$$
v\left(t, \tau, x ; v_{\tau}\right) \leq u\left(t, \tau, x ; u_{\tau}\right) .
$$

If $V\left(t, \tau, x ; u_{\tau}\right)$ is a super solution of the Cauchy problem of (3.1) with initial condition $V_{\tau}, u_{\tau} \leq V_{\tau}$ we obtain, by analogous arguments

$$
u\left(t, \tau, x ; u_{\tau}\right) \leq V\left(t, \tau, x ; V_{\tau}\right) \text {, a.e. in }[\tau, T] \times \Omega .
$$

Since the estimates above are uniform in a bounded set containing the initial conditions, we may extend the result to the interval $[T,(2 T-\tau)]$ and, by iteration, we can complete the proof of the theorem.

From Theorem 5.2, we immediately obtain invariance of some subsets of $L^{\infty}(\Omega)$ (or the functions in $L^{\infty}\left(\mathbb{R}^{N}\right)$ vanishing outside $\Omega$, with the obvious identification) under the process $S(t, \tau)$.

Corollary 5.3. Suppose $f(t, x)=f(x)$ and $h(t, x)=h(x)$ satisfy the hypotheses of Theorem 5.2 and are independent of $t$. If $v(V)$ is an non negative (non positive) equilibrium of (3.1) with $f(h)$ substituted for $g$ then the set

$$
U=\left\{u \in L^{\infty}(\Omega) ; v \leq u \leq V\right\} \quad\left(U=\left\{u \in C^{0}\left(\mathbb{R}^{N}\right) ; v \leq u \leq 0\right\}\right)
$$

is positively invariant under the process $S(t, \tau)$, that is $S(t, \tau) U \subset U$ for all $t \geq \tau$. 


\section{UPPER SEMI-CONTINUITY OF THE PULlBACK ATTRACTORS}

〈Sec6) A natural question is the dependence of the pullback attractors of (3.1) upon parameters. Suppose, for instance, that we have a family of functions $g_{\beta}(t, x)$, with $\beta$ in some normed space, $g_{\beta}(t, x) \rightarrow g_{\beta_{0}}(t, x)$ as $\beta \rightarrow \beta_{0}$ and denote by $S_{\beta}(t, \tau) u_{\beta}(\tau, x)=u_{\beta}\left(t, \tau, x ; u_{\tau}\right)$ the evolution process associated with the problem (3.1) with $g$ replaced by $g_{\beta}$. Under suitable hypotheses, we prove the upper semi-continuity of the pullback attractors as $\beta \rightarrow \beta_{0}$, for each $t \in \mathbb{R}$ that is, we show that

$$
\operatorname{dist}\left(\mathcal{A}_{\beta}(t), \mathcal{A}_{\beta_{0}}(t)\right) \rightarrow 0 \quad \text { as } \quad \beta \rightarrow \beta_{0} \text { in } L^{\infty}(\mathbb{R})
$$

where $\left\{A_{\beta}(t) ; t \in \mathbb{R}\right\}$ denotes the pullback attractor of $S_{\beta}(t, \tau)$ in $X$, and $\operatorname{dist}\left(\mathcal{A}_{\beta}(t), \mathcal{A}_{\beta_{0}}(t)\right)$ is the Hausdorff semi-distance in $L^{p}(\Omega)$ defined by (2.1), for each $t \in \mathbb{R}$.

〈TheoUpper01〉 Theorem 6.1. Assume the same hypotheses of Lemma 4.1, with $g$ replaced by $g_{\beta}$ for $\beta$ in a neighborhood of $\beta_{0}$. Suppose additionally that the Lipschitz constants of the functions $g_{\beta}$ in bounded sets of $\mathbb{R} \times \mathbb{R}$ can be chosen independently of $\beta$ and $g_{\beta}(t, x) \rightarrow g_{\beta_{0}}(t, x)$ as $\beta \rightarrow \beta_{0}$ uniformly in bounded sets of $\mathbb{R} \times \mathbb{R}$. Then $S_{\beta}(t, \tau)\left(u_{\tau}\right) \rightarrow S_{\beta_{0}}(t, \tau)\left(u_{\tau}\right)$ in the $L^{p}(\Omega)-$ norm, $1 \leq p \leq \infty$, uniformly for $t \in[\tau, c]$ with $c<\infty$ and $u_{\tau}$ in a bounded subset of $X$.

Proof. Let $u_{\beta}\left(t, \tau, x ; u_{\tau}\right)=S_{\beta}(t, \tau) u_{\beta}(\tau, x)$ and $u_{\beta_{0}}\left(t, \tau, x ; u_{\tau}\right)=S_{\beta_{0}}(t, \tau) u_{\beta}(\tau, x)$ be the solutions of (3.1) with initial condition $u_{\tau}$, and parameters $\beta$ and $\beta_{0}$, respectively, Then

$$
\begin{aligned}
& \left(u_{\beta}-u_{\beta_{0}}\right)\left(t, \tau, x ; u_{\tau}\right) \\
= & \left.\int_{\tau}^{t} e^{-(t-s)}\left[g_{\beta}\left(s, K u_{\beta}\left(s, \tau, x ; u_{\tau}\right)\right)-g_{\beta_{0}}\left(s, K u_{\beta_{0}}\right)\left(s, \tau, x ; u_{\tau}\right)\right)\right] d s .
\end{aligned}
$$

Summing and subtracting the term $\int_{\tau}^{t} e^{-(t-s)} g_{\beta}\left(s, K u_{\beta_{0}}\left(s, \tau, x ; u_{\tau}\right)\right) d s$, we get

$$
\begin{aligned}
& \left(u_{\beta}-u_{\beta_{0}}\right)\left(t, \tau, x ; u_{\tau}\right) \\
= & \int_{\tau}^{t} e^{-(t-s)}\left[g_{\beta}\left(s, K u_{\beta}\left(s, \tau, x ; u_{\tau}\right)\right)-g_{\beta}\left(s, K u_{\beta_{0}}\left(s, \tau, x ; u_{\tau}\right)\right)\right] d s \\
+ & \int_{\tau}^{t} e^{-(t-s)}\left[g_{\beta}\left(s, K u_{\beta_{0}}\left(s, \tau, x ; u_{\tau}\right)\right)-g_{\beta_{0}}\left(s, K u_{\beta_{0}}\left(s, \tau, x ; u_{\tau}\right)\right)\right] d s
\end{aligned}
$$

for any $t \geq \tau$ and $x \in \Omega$.

From estimates (4.1) and (3.5) it follows that $K u_{\beta}\left(s, \tau, x ; u_{\tau}\right)$ and $K u_{\beta_{0}}\left(s, \tau, x ; u_{\tau}\right)$ remain in a bounded set $B \subset \mathbb{R}$ for any $\tau \leq s \leq t$ and $x \in \Omega$. If $k_{B}$ denotes the Lipschitz constant of $g_{\beta}$ in $[\tau, t] \times B$ and

$$
\left\|g_{\beta}-g_{\beta_{0}}\right\|_{\infty}:=\sup \left\{\mid g_{\beta}(s, x)-g_{\beta_{0}}(s, x) ; \quad(s, x) \in[\tau, t] \times B\right\},
$$

we obtain, using (3.4) 


$$
\begin{aligned}
& \left\|\left(u_{\beta}-u_{\beta_{0}}\right)\left(t, \tau, \cdot ; u_{\tau}\right)\right\|_{X} \\
\leq & \int_{\tau}^{t} e^{-(t-s)}\left\|g_{\beta}\left(s, K u_{\beta}\left(s, \tau, \cdot ; u_{\tau}\right)\right)-g_{\beta}\left(s, K u_{\beta_{0}}\left(s, \tau, \cdot ; u_{\tau}\right)\right)\right\| d s \\
+ & \int_{\tau}^{t} e^{-(t-s)}\left\|g_{\beta}\left(s, K u_{\beta_{0}}\left(s, \tau, \cdot ; u_{\tau}\right)\right)-g_{\beta_{0}}\left(s, K u_{\beta_{0}}\left(s, \tau, \cdot ; u_{\tau}\right)\right)\right\| d s \\
\leq & k_{B} \int_{\tau}^{t} e^{-(t-s)}\left\|K u_{\beta}\left(s, \tau, \cdot ; u_{\tau}\right)-K u_{\beta_{0}}\left(s, \tau, \cdot ; u_{\tau}\right)\right\| d s \\
+ & \left\|g_{\beta}-g_{\beta_{0}}\right\|_{\infty}|\Omega|^{\frac{1}{p}} \int_{\tau}^{t} e^{-(t-s)} d s \\
\leq & k_{B} \int_{\tau}^{t} e^{-(t-s)}\left\|u_{\beta}\left(s, \tau, \cdot ; u_{\tau}\right)-u_{\beta_{0}}\left(s, \tau, \cdot ; u_{\tau}\right)\right\| d s \\
+ & \left\|g_{\beta}-g_{\beta_{0}}\right\|_{\infty}|\Omega|^{\frac{1}{p}}
\end{aligned}
$$

where we have indicated by $\|\cdot\|$ the norm in the space $X=L^{p}(\Omega)$.

By Gronwall's inequality, it follows that

$$
\left\|\left(u_{\beta}-u_{\beta_{0}}\right)\left(t, \tau, \cdot ; u_{\tau}\right)\right\|_{X} \leq|\Omega|^{\frac{1}{p}}\left\|g_{\beta}-g_{\beta_{0}}\right\|_{\infty} e^{k_{B}(t-\tau)}
$$

concluding the proof.

Theorem 6.2. Under the same conditions of Theorem 6.1, the family of pullback attractors $\left\{\mathcal{A}_{\beta}(t) ; t \in \mathbb{R}\right\}$ is upper semicontinuous at $\beta=\beta_{0}$, at each $t \in \mathbb{R}$.

Proof. Denote by $M$ the radius of an absorbing ball for the process $S_{\beta}$ for $\beta$ close to $\beta_{0}$, whose existence is guaranteed by Lemma 4.1. Given $\delta>0$, let $\tau \in \mathbb{R}$ such that $\operatorname{dist}\left(S_{\beta_{0}}(t, \tau) B(0 ; M), \mathcal{A}_{\beta_{0}}(t)\right)<\delta / 2$. By Theorem 6.1

$$
\left\|\left(u_{\beta}-u_{\beta_{0}}\right)\left(t, \tau, \cdot ; u_{\tau}\right)\right\|_{X} \rightarrow 0
$$

as $\beta \rightarrow \beta_{0}$ uniformly for $u_{\tau}$ in bounded subsets of $X$. Since $\bigcup_{s \in \mathbb{R}} \mathcal{A}_{\beta}(s) \subset B(0 ; M)$, for $\beta$ in a neighborhood of $\beta_{0}$, there exists $r_{0}>0$ such that

$$
\sup _{a_{\beta} \in \mathcal{A}_{\beta}(\tau)}\left\|S_{\beta}(t, \tau) a_{\beta}-S_{\beta_{0}}(t, \tau) a_{\beta}\right\|_{X}<\delta / 2
$$

for all $\beta \in B\left(\beta_{0}, r_{0}\right)$, the ball centered at $\beta_{0}$ of radius $r_{0}>0$ in $L^{\infty}(\mathbb{R})$.

Then

$$
\begin{aligned}
& \operatorname{dist}\left(\mathcal{A}_{\beta}(t), \mathcal{A}_{\beta_{0}}(t)\right) \\
\leq & \operatorname{dist}\left(S_{\beta}(t, \tau) \mathcal{A}_{\beta}(\tau), S_{\beta_{0}}(t, \tau) \mathcal{A}_{\beta}(\tau)\right)+\operatorname{dist}\left(S_{\beta_{0}}(t, \tau) \mathcal{A}_{\beta}(\tau), S_{\beta_{0}}(t, \tau) \mathcal{A}_{\beta_{0}}(\tau)\right) \\
= & \sup _{a_{\beta} \in \mathcal{A}_{\beta}(\tau)} \operatorname{dist}\left(S_{\beta}(t, \tau) a_{\beta}, S_{\beta_{0}}(t, \tau) a_{\beta}\right)+\operatorname{dist}\left(S_{\beta_{0}}(t, \tau) \mathcal{A}_{\beta}(\tau), \mathcal{A}_{\beta_{0}}(t)\right) \\
< & \frac{\delta}{2}+\frac{\delta}{2}
\end{aligned}
$$

and the upper semicontinuity is proved. 


\section{THE FORWARD ASYMPTOTICALLY AUTONOMOUS CASE}

$\langle\mathrm{NS}\rangle$

We now consider the forward dynamics of (3.1) in the case where $g(t, x)$ converges to a function $g_{0}(x)$ independent of $t$, when $t \rightarrow \infty$. Then (with appropriate hypotheses), the process generated by (3.1) becomes an asymptotically autonomous process, which is defined as follows (see, for example [11]).

Definition 7.1. An evolution process $\{S(t, \tau) ; t \geq \tau \in \mathbb{R}\}$ in a Banach space $X$ is called asymptotically autonomous with limit (autonomous) process $\left\{S_{0}(t) ; t \geq 0\right\}$ if

$$
S\left(t_{j}+\tau_{j}, \tau_{j}\right) x_{j} \rightarrow S_{0}(t) x
$$

for any three sequences $t_{j} \rightarrow t, \tau_{j} \rightarrow \infty, x_{j} \rightarrow x$, as $j \rightarrow \infty$, with $x, x_{j} \in X, 0 \leq t, t_{j}<\infty$, and $\tau_{j} \geq t_{0}$.

Consider the (autonomous) problem in $X=L^{p}(\Omega), 1 \leq p \leq \infty$.

$$
\left\{\begin{array}{l}
\partial_{t} u(t, x)=-u(t, x)+g_{0}(K u(t, x)) \text { for } t \geq \tau \in \mathbb{R} \text { and } x \in \Omega, \\
u(\tau, \cdot)=u_{\tau}(\cdot) \text { in } \Omega, \\
u(t, x)=0 \text { for } t \geq \tau \in \mathbb{R} \text { and } x \in \mathbb{R}^{N} \backslash \Omega
\end{array}\right.
$$

Lemma 7.2. Suppose $g(t, x)$ and $g_{0}$ satisfy the hypotheses required for $g$ in Lemma 4.1 and $g(t, x) \rightarrow g_{0}(x)$ as $t \rightarrow \infty$, uniformly for $x$ in bounded sets of $\mathbb{R}$. Then the process $\{S(t, \tau) ; t \geq \tau \in \mathbb{R}\}$ generated by (3.1) is asymptotically autonomous with limit autonomous process $\left\{S_{0}(t, \tau) ; t \geq \tau \in \mathbb{R}\right\}$ generated by (7.1).

Proof. Suppose $t_{j} \rightarrow t, \tau_{j} \rightarrow \infty, u_{j} \rightarrow u$ in $X$, as $j \rightarrow \infty$, with $u, u_{j} \in X, t \geq 0$, and $t_{j}<\infty$.

Then

$$
\begin{aligned}
S\left(t_{j}+\tau_{j}, \tau_{j}\right) u_{j}-S_{0}\left(t+\tau_{j}, \tau_{j}\right) u & =\left(S\left(t_{j}+\tau_{j}, \tau_{j}\right) u_{j}-S\left(t+\tau_{j}, \tau_{j}\right) u_{j}\right)+ \\
\left.\left.(7.2) \text { asympt(tatt } \tau_{j}, \tau_{j}\right) u_{j}-S\left(t+\tau_{j}, \tau_{j}\right) u\right) & +\left(S\left(t+\tau_{j}, \tau_{j}\right) u-S_{0}\left(t+\tau_{j}, \tau_{j}\right) u\right) .
\end{aligned}
$$

If $t_{j} \rightarrow t$, the first term to the right in (7.2) goes to zero by continuity of the semigroup. The second term also goes to zero, as $u_{j} \rightarrow u$ by continuity of the semigroup with respect to initial conditions. This can be proved in our case, as follows: by Lemma 4.1, $S\left(s+\tau_{j}, \tau_{j}\right) u$ belongs to a bounded set in $L^{p}(\Omega)$, for $s, \tau_{j} \in[0, \infty)$ and $u$ a bounded set in $L^{p}(\Omega)$. Therefore, from estimate (3.5),$K S\left(s+\tau_{j}, \tau_{j}\right) u_{j}$ and $K S_{0}\left(s+\tau_{j}, \tau_{j}\right) u$ belong to a bounded set in $L^{\infty}(\Omega)$, for $s, \tau_{j} \in[0, \infty$. Then, using that $g$ is locally Lipschitz and Gronwall's inequality, we obtain that $S\left(t+\tau_{j}, \tau_{j}\right) u_{j} \rightarrow S\left(t+\tau_{j}, \tau_{j}\right) u$ as claimed.

For the last term, we may use results of Section [6. Indeed, defining, for $\beta \in \mathbb{R}$, the function $g_{\beta}(t, x)=g(t+\beta, x)$, it follows from the assumptions that $g_{\beta}(t, x) \rightarrow g_{0}(x)$ as $\beta \rightarrow \infty$, uniformly in bounded sets of $\mathbb{R} \times \mathbb{R}$. Denoting by $S_{\beta}(t, \tau)$ the semigroup generated by (1.1), with $g_{\beta}$ substituted for $g$, we obtain from Theorem 6.1, that $S_{\beta}(t, \tau) u \rightarrow S_{0}(t, \tau) u$ as $\tau \rightarrow \infty$. Since $S_{\tau_{j}}(t, \tau) u=S\left(t+\tau_{j}, \tau\right) u$, the claimed convergence is proved. 
We now obtain some results concerning the convergence of solutions of (3.1) to equilibria of (7.1). Similar results were obtained in ([8]) and ([11]), but we present here a more direct approach for our specific problem, based on the properties of an "energy functional" in $X$.

To define this functional we need the following additional hypothesis on the function $g_{0}$ :

(H1) There exists a constant $a>0$ such that

$$
\left|g_{0}(s)\right| \leq a \text { for all } s \in \mathbb{R} .
$$

(H2) The function $g_{0}$ is strictly increasing and the function

$$
f(s)=-\frac{1}{2} s^{2}-i(s), \quad s \in[-a, a]
$$

where $i$ is given by

$$
i(s)=-\int_{0}^{s} g_{0}^{-1}(\theta) d \theta, \quad s \in[-a, a]
$$

has a global minimum

We then define the functional in $Y:=\{u \in X ;|u(x)|<a$, for all $x \in \Omega\}$.

$$
\begin{aligned}
\mathcal{L}(u) & =\int_{\mathbb{R}^{n}}[f(u(x))-f(\bar{u})] d x+\frac{1}{4} \int_{\mathbb{R}^{n}} \int_{\mathbb{R}^{n}} J(x, y)[u(x)-u(y)]^{2} d x d y \\
& =\int_{\Omega}[f(u(x))-f(\bar{u})] d x+\frac{1}{4} \int_{\Omega} \int_{\Omega} J(x, y)[u(x)-u(y)]^{2} d x d y
\end{aligned}
$$

where $\bar{u}$ is a global minimum of $f$.

Remark 7.3. If $|g(t, x)| \leq a$, for $(t, x) \in \mathbb{R} \times \mathbb{R}$ the hypotheses of Lemma 4.1 hold with $k_{1}=0$ and $k_{2}=a$. It follows then, from estimate (4.1), that the set $Y$ is (pullback and forward) absorbing and, therefore, positively invariant for the process generated by (3.1). Also, since $g(t, x) \rightarrow g_{0}(x)$ uniformly in bounded sets, this property holds for $x$ in a bounded set, if $t$ is sufficiently large. Therefore, if we are interested only in solutions in $Y$, we may suppose that it holds for all $x$ by doing an appropriate "cut-off" of $g(t, \cdot)$, if necessary.

Proposition 7.4. Under hypotheses (H1) and (H2), the functional $\mathcal{L}$ is continuous (with respect to the $L^{p}$ norm).

Proof. Note that, given $u \in Y$, as $|u(x)| \leq a$, for all $x \in \Omega$ there exists a positive constant $f_{0}$ such that

$$
|f(u(x))-f(\bar{u})| \leq|f(u(x))|+|f(\bar{u})| \leq f_{0}, \text { for almost every (a.e.) } x \in \Omega .
$$

Let $\left(u_{n}\right)$ a sequence converging to $u$ in the norm of $L^{p}(\Omega)$. Then, by Theorem 4.9 in [2], we can extract a subsequence $\left(u_{n_{k}}\right)$, such that, $u_{n_{k}}(x) \rightarrow u(x)$ a.e. in $\Omega$. Since from (H2), it follows that $f$ is continuous, $f\left(u_{n_{k}}(x)\right) \rightarrow f(u(x))$ a.e. Thus

$$
\lim _{k \rightarrow \infty}\left[f\left(u_{n_{k}}(x)\right)-f(\bar{u})\right]=[f(u(x))-f(\bar{u})] \text {, a.e. }
$$

and

$$
\lim _{k \rightarrow \infty}\left[u_{n_{k}}(x)-u_{n_{k}}(y)\right]^{2}=[u(x)-u(y)]^{2}, \text { a.e. }
$$


Now, we write

$$
\mathcal{L}(u)=\mathcal{L}_{1}(u)+\mathcal{L}_{2}(u)
$$

where

$$
\mathcal{L}_{1}(u)=\int_{\Omega}[f(u(x))-f(\bar{u})] d x
$$

and

$$
\mathcal{L}_{2}(u)=\frac{1}{4} \int_{\Omega} \int_{\Omega} J(x, y)[u(x)-u(y)]^{2} d x d y .
$$

Since

$$
\left|f\left(u_{n_{k}}(x)\right)-f(\bar{u})\right| \leq f_{0} \in L^{1}(\Omega),
$$

by Lebesgue's dominated convergence theorem (see [2]), we have

$$
\lim _{k \rightarrow \infty} \mathcal{L}_{1}\left(u_{n_{k}}\right)=\mathcal{L}_{1}(u)
$$

Analogously

$$
\left|u_{n_{k}}(x)-u_{n_{k}}(y)\right|^{2} \leq 4 a^{2} \in L^{1}(\Omega) .
$$

Hence, also by Lebesgue's dominated convergence theorem, we obtain

$$
\lim _{k \rightarrow \infty} \mathcal{L}_{2}\left(u_{n_{k}}\right)=\mathcal{L}_{2}(u)
$$

Therefore, from (7.4) and (17.5), it follows that

$$
\lim _{k \rightarrow \infty} \mathcal{L}\left(u_{n_{k}}\right)=\mathcal{L}(u)
$$

Thus $\left(\mathcal{L}\left(u_{n}\right)\right)$ is a sequence in $\mathbb{R}$ such that every subsequence has a subsequence that converges to $\mathcal{L}(u)$, and we obtain

$$
\lim _{n \rightarrow \infty} \mathcal{L}\left(u_{n}\right)=\mathcal{L}(u)
$$

Remark 7.5. The integrand in the functional $\mathcal{L}$ given in (7.3) is always non negative since $J$ is positive and $\bar{u}$ is a global minimum of $f$. Thus $\mathcal{L}$ is lower bounded.

As shown in [1] $\mathcal{L}$ is a Lyapunov functional for dynamical system $\left\{S_{0}(t) ; t \geq 0\right\}$ generated by (7.1) in $L^{2}(\Omega)$, with derivative along the solutions $u\left(t, \tau, \cdot ; u_{\tau}\right)$ given by

where

$$
\frac{d}{d t} \mathcal{L}\left(u\left(t, \tau, \cdot ; u_{\tau}\right)=-I\left(u\left(t, \tau, \cdot ; u_{\tau}\right)\right)\right.
$$

$I\left(u\left(t, \tau, \cdot ; u_{\tau}\right)\right)=\int_{\Omega}\left[K u\left(t, \tau, x ; u_{\tau}\right)-g_{0}^{-1}\left(u\left(t, \tau, x ; u_{\tau}\right)\right)\right]\left[-u\left(t, \tau, x ; u_{\tau}\right)+g_{0}\left(K u\left(t, x ; \tau, u_{\tau}\right)\right)\right] d x$

In the nonautonomous case, we can obtain a similar result, with an additional term which is small for $t$ big. The proof is also analogous to the one obtained in [1] for the autonomous case and will be omitted. 
Theorem 7.6. Suppose that the hypotheses (H1) and (H2) hold and the functions $g(t, \cdot)$ are invertible, for all $t \in \mathbb{R}$. Let $u\left(t, \tau, \cdot ; u_{\tau}\right)$ be a solution of (3.1) with $u\left(t, \tau, x ; u_{\tau}\right) \leq a$. Then $\mathcal{L}\left(u\left(t, \tau, \cdot ; u_{\tau}\right)\right)$ is differentiable with respect to $t$ for $t>\tau$ and, for any $u \in L^{2}\left(\mathbb{R}^{N}\right)$,

$$
\frac{d}{d t} \mathcal{L}\left(u\left(t, \tau, \cdot ; u_{\tau}\right)=-I\left(u\left(t, \tau, \cdot ; u_{\tau}\right)\right)+R\left(t, u\left(t, \tau, \cdot ; u_{\tau}\right)\right)\right.
$$

where

$$
\begin{aligned}
I\left(u\left(t, \tau, \cdot ; u_{\tau}\right)\right) & =\int_{\Omega}\left[K u\left(t, \tau, x ; u_{\tau}\right)-g_{0}^{-1}\left(u\left(t, \tau, x ; u_{\tau}\right)\right)\right]\left[-u\left(t, \tau, x ; u_{\tau}\right)\right. \\
& \left.+g_{0}\left(K u\left(t, x ; \tau, u_{\tau}\right)\right)\right] d x \\
R\left(t, u\left(t, \tau, \cdot ; u_{\tau}\right)\right) & =\int_{\Omega}\left[g^{-1}\left(t, u\left(t, \tau, x ; u_{\tau}\right)\right)-g_{0}^{-1}\left(u\left(t, \tau, x ; u_{\tau}\right)\right)\right]\left[-u\left(t, \tau, x ; u_{\tau}\right)\right. \\
& \left.+g_{0}\left(K u\left(t, \tau, x ; u_{\tau}\right)\right)\right] d x
\end{aligned}
$$

Furthermore, $I\left(u\left(t, \tau, \cdot ; u_{\tau}\right)\right)=0$ if and only if $u\left(t, \tau, \cdot ; u_{\tau}\right)$ is one of the equilibria of the autonomous system (7.1).

Remark 7.7. If $u\left(t, \tau, \cdot ; u_{\tau}\right)$ remains bounded, the second term $R\left(t, u\left(t, \tau, \cdot ; u_{\tau}\right)\right)$ in the expression above for the derivative of $\mathcal{L}\left(u\left(t, \tau, \cdot ; u_{\tau}\right)\right)$ goes to zero, as $t \rightarrow \infty$ and, therefore, the derivative along the solution of the nonautonomous system approaches $-I$, which is the derivative along a solution of the autonomous system.

Lemma 7.8. The forward orbits of the process $\{S(t, \tau) ; t \geq \tau \in \mathbb{R}\}$ generated by (3.1) are precompact in $X$.

Proof. Follows from the existence of attractors, or may be proved using the variation of constants formula and the decomposition $S(t, \tau) u_{\tau}=T(t, \tau) u_{\tau}+U(t, \tau) u_{\tau}$ given in Theorem 4.2 .

$\left\langle\right.$ lasalle ${ }^{2}$ Theorem 7.9. Suppose that $\mathcal{L}$ assumes a finite number of values in the set $E$ of equilibria of the dynamical system (7.1). If $u\left(t, \tau, x ; u_{\tau}\right)$ is a solution of (3.1) then

$$
u\left(t, \tau, x ; u_{\tau}\right) \rightarrow \mathcal{M} \text {, as } t \rightarrow+\infty,
$$

where $\mathcal{M}$ is a level set of the functional $\mathcal{L}$ restricted to $E$.

Proof. Let $\mathcal{O}=S(t, \tau) u_{\tau}$ denote the forward orbit of $u_{\tau}$ and $E_{1}, E_{2}, \ldots, E_{m}$ the level sets of $\mathcal{L}$ in $E$, in ascending order. Observe that $\overline{\mathcal{O}}$ is positively invariant and compact.

Write $\tilde{E}_{j}=E_{j} \cap \overline{\mathcal{O}}, \quad i=1,2, \ldots, m, \tilde{E}=E \cap \overline{\mathcal{O}}=\cup_{j=1}^{m} \tilde{E}_{j}$. Then, each $\tilde{E}_{j}$ is compact, since $E_{j}$ is closed. We first claim that $\tilde{E}$ is not empty. In fact, if this were true, then $I<0$ in the compact set $\overline{\mathcal{O}}$ implies $I<-m$, for some positive number $m$ and then $\frac{d}{d t} \mathcal{L}\left(S(t, \tau) u_{\tau}\right)<$ $-m / 2$ in $\overline{\mathcal{O}}$ for $t$ big enough and, a fortiori, also in $\mathcal{O}$. But this is impossible, since $\mathcal{L}$ is bounded below.

Let then $\tilde{E}_{k}$ be the minimum level which intercepts $\overline{\mathcal{O}}$. We now show that no other level is in the $\Omega$-limit of $\mathcal{O}$. For this, choose a neighborhood $V_{i}$ of each $\tilde{E}_{i}$, such that $\max \left\{\mathcal{L}(u): u \in V_{i}\right\}<\min \left\{\mathcal{L}(u): u \in V_{i+1}\right\}, i=1,2, \ldots, m-1$. Let $\mathcal{L}_{k}$ be the value 
of $\mathcal{L}$ in $\tilde{E}_{k}$ and $W_{\varepsilon}^{i}=\left\{u \in V_{i}: \mathcal{L}_{i}-\varepsilon<\mathcal{L}(u)<\mathcal{L}_{i}+\varepsilon\right\}, i=1,2, \ldots, m-1$, with $\varepsilon$ chosen in such a way that $\overline{W_{\varepsilon}^{i}} \subset V_{i}$. Reasoning again by compactness we may find $\bar{t}$ such that $\frac{d}{d t} \mathcal{L}\left(S(t, \tau) u_{\tau}\right)<0$ in $\overline{\mathcal{O}}-\cup_{i} W_{\varepsilon}^{i}$, if $t \geq \bar{t}$. Now, if $s>\bar{t}$ is such that $S(s, \tau) u_{\tau}$ belongs to $W_{\varepsilon}^{k}$ (such an $s$ exists, since the $\Omega$-limit set intersects $E_{k}$ ), then $S(t, \tau) u_{\tau}$ remains in $W_{\varepsilon}^{k}$ for all $t>s$. In fact, $\mathcal{L}\left(S(t, \tau) u_{\tau}\right)$ cannot become bigger than $\mathcal{L}_{k}+\varepsilon$, since it decreases in $V_{i} \backslash W_{\varepsilon}^{i}$. It cannot also become smaller than $\mathcal{L}_{k}-\varepsilon$. In fact in this case, $S(t, \tau) u_{\tau}$ will never enter any of the neighborhoods $\cup_{i} W_{i}$ again since $\mathcal{L}\left(S(t, \tau) u_{\tau}\right)$ decreases in $\overline{\mathcal{O}}-\cup_{i} W_{\varepsilon}^{i}$. This is a contradiction, since it must return to $W_{\varepsilon}^{k}$.

Therefore, we must have $S(t, \tau) u_{\tau} \subset W_{\varepsilon}^{k}$ for $t$ big enough. Since $\varepsilon$ is arbitrary, we must have $\cap_{t \geq \tau} \cup_{s \geq t} \overline{S(s, \tau)\left(u_{\tau}\right)} \subset E_{k}$, as claimed

Corollary 7.10. Suppose, that (7.1) has a finite number of equilibria in each level set of $\mathcal{L}$. Then any solution $u\left(t, \tau, x ; u_{\tau}\right)$ of (3.1) converges to a single equilibrium of (7.1).

Proof. We must show that the $\Omega$-limit of $u\left(t, \tau, x ; u_{\tau}\right)$ is a single equilibrium. From Theorem 7.9 it follows that it must be a subset of the set of $E$ equilibria of (7.1) and also contained in a level set of $\mathcal{L}$. Since it must also be connected, the result follows immediately.

\section{REFERENCES}

FAH [1] F. D. M. Bezerra, A. L. Pereira and S. H. Da Silva, Existence and continuity of global attractors and nonhomogeneous equilibria for a class of evolution equation with non local terms. J. Math. Anal. Appl., 396 (2012) 590-600.

Brezis [2] H. Brezis, Functional Analysis, Sobolev Spaces and Partial Differential Equations. Springer, New York, 1984.

Carvalho [3] A. N. Carvalho, J. A. Langa, J. C. Robinson, On the continuity of pullback attractors for evolution processes. Nonlinear Analysis, 71 (2009) 1812-1824.

CLR [4] A. N. Carvalho, J. A. Langa, J. C. Robinson, Attractors for Infinite-dimensional Non-autonomous Dynamical Systems. Applied Mathematical Sciences 182, Springer-Verlag, 2012.

CS [5] A. N. Carvalho, S. Sonner, Pullback exponential attractors for evolution process in Banach spaces: theoretical results. Communications on Pure and Applied Analysis, 12, 6, 2013.

CV [6] V. V. Chepyzhov, M. I. Vishik, Attractors for Equations of Mathematical Physics, in: Colloquium Publications, vol. 49, American Mathematical Society, 2002.

DK [7] J. L. Daleckii and M. G. Krein, Stability of Solutions of Differential Equations in Banach Spaces. American Mathematical Society, Providence, Rhode Island, 1974.

polacik [8] J. Foldes, P. Polacik, Convergence to a steady state for asymptotically autonomous semilinear heat equations in $\mathbb{R}^{n}$. J. Diffferential Equations 2517 (2011), 1903-1922.

KI [9] P. E. Kloeden, Pullback attractors in nonautonomous difference equations. J. Differ. Equations Appl. 6 (1) (2000) 33-52.

ladas [10] G. E. Ladas and V. Lakshmikantham, Differential equations in abstract spaces, Academic Press, N.Y., 1972.

mischaikow [11] K. Mischaikow, H. Smith and H. R. Thieme, Asymptotically autonomous semiflows: chain recurrence and Lyapunov functions, Trans. AMS, 347 (5) (1995), 1669 - 1685.

Sch [12] B. Schmalfuss, Attractors for the non-autonomous dynamical systems. International Conference on Differential Equations, Vol. 1, 2 (Berlin, 1999), 684-689, World Sci. Publishing, River Edge, NJ, 2000.

Rall [13] L. B. Rall, Nonlinear Functional Analysis and Applications. Academic Press, New York-London, 1971. 
Sell [14] G. R. Sell, Non-autonomous differential equations and dynamical systems. Trans. Amer. Math. Soc., 127 (1967) 241-283.

T [15] Teman, R., Infinite Dimensional Dynamical Systems in Mechanics and Physics. Springer, 1988.

(F. D. M. Bezerra) Departamento de Matemática, Universidade Federal da Paraíba, Cidade Universitária-Campus I, 58051-900 JoÃo Pessoa PB, Brazil.

E-mail address: flank@mat.ufpb.br

(S. H. Da Silva) Unidade Acadêmica de Matemática, Universidade Federal de Campina Grande, 58051-900 Campina Grande PB, Brazil.

E-mail address: horacio@dme.ufcg.edu.br

(A. L. Pereira) Universidade de São Paulo, Instituto de Matemática e Estatística, 13565-905 SÃo Paulo SP, Brazil.

E-mail address: alpereir@ime.usp.br 